\title{
PARALLEL UNSTRUCTURED MESH ADAPTATION BASED ON ITERATIVE REMESHING AND REPARTITIONING
}

\author{
Luca Cirrottola $^{1 *}$, Algiane Froehly ${ }^{2}$ \\ ${ }^{1}$ INRIA, Université de Bordeaux, CNRS, Bordeaux INP, IMB UMR 5251, 200 Avenue de la Vieille \\ Tour, 33405 Talence cedex, France, luca.cirrottola@inria.fr \\ ${ }^{2}$ INRIA Direction Générale Déléguée à l'Innovation, InriaSoft, Mmg Consortium, \\ algiane.froehlyeinria.fr
}

Key words: Parallel mesh adaptation, unstructured meshes, mesh migration

\begin{abstract}
We present a parallel unstructured mesh adaptation algorithm based on iterative remeshing and mesh repartitioning. The algorithm rests on a two-level parallelization scheme allowing to tweak the mesh group size for remeshing, and on a mesh repartitioning scheme based on interface displacement by front advancement. The numerical procedure is implemented in the open source ParMmg software package. It enables the reuse of existing sequential remeshing libraries, a non-intrusive linkage with thirdparty solvers, and a tunable exploitation of distributed parallel environments. We show the efficiency of the approach by comparing interface displacement repartitioning with graph-based repartitioning, and by showing isotropic weak-scaling tests and preliminary anisotropic tests.
\end{abstract}

\section{INTRODUCTION}

Modern computational mechanics solvers nowadays routinely exploit parallel, distributed memory computer architectures. Even if a parallel solver could in principle use a sequential remesher by gathering the distributed mesh on a single process, due to memory limitations it is not always possible to guarantee that a large distributed mesh can be gathered on a single computing node. Beside this primary feasibility consideration, sequential remeshing in a parallel simulation also represents a significant performance bottleneck [1]. Parallel remeshing is thus becoming increasingly demanded.

Among the many previous works on parallel remeshing (for example [2] [3] [4] [5] [6] [7] [8] [9]), a broad classification can be made into methods that aim at parallelizing the remeshing kernel itself, and methods that aim at employing a sequential remeshing kernel in a parallel framework by leveraging a parallel repartitioning phase. We have opted for a modular approach by adopting an iterative remeshingrepartitioning scheme that does not modify the adaptation kernel [9], allowing the reuse of an existing sequential remeshing kernel. Our method is implemented into the parallel ParMmg application and library [10], built on top of the sequential Mmg3d remesher [11] for tetrahedral mesh adaptation. Both software are free and open source.

The rest of this paper is organized as follows. Section 2 briefly recalls the parallelization algorithm, which is detailed in [12]. Section 3 discusses the parallel performances at the current level of implementation, while section 4 sums up our observations and future research lines. 


\section{METHOD}

As described in depth in [12], at each iteration of the parallel algorithm the sequential remeshing kernel is applied on the interior partition on each process, while maintaining fixed (non-adapted) parallel interfaces. Then the adapted mesh is repartitioned in order to move the non-adapted frontiers to the interior of the partitions at the next iteration, thus eliminating the presence of non-adapted zones as the iterations progress. Although the repartitioning step can be accomplished through standard mesh partitioning libraries, differently from [9] we have explored the usage of a direct parallel interface displacement method (or diffusion algorithm) to explicitly prioritize interface displacement over load balancing.

\section{PARALLEL PERFORMANCE ASSESSMENT}

We show the performances of the parallel algorithm on two academic edge sizemaps, by means of a weak scaling and a strong scaling test. Both the weak and strong scaling tests have been performed with the release v1.3.0 of ParMmg[10].

\subsection{Weak scaling}

A weak scaling test is shown in table 1. A sphere of radius 10 is uniformly refined while keeping the workload of each process as constant as possible as the number of processes is increased. The test is performed on the bora nodes of the PIaERIM cluster ${ }^{1}$. The weak scaling performances are shown in figure 1a. The slow increase in the time spent in the repartitioning and redistribution phase still leaves space for some implemen the preliminary results sh than 64 cores. This impro of the pair of processes interacting presence of data to exchange whose cost grew quadratically.

\section{Register for free at https//www.scipedia.com to download the version without the watermark 3.2 Strong scaling}

A strong scaling test is performed with an isotropic sizemap $h$ describing a double Archimedean spiral

$$
h(x, y)=\min \left(1.6+\left|\rho-a \theta_{1}\right|+0.005,1.6+\left|\rho+a \theta_{2}\right|+0.0125\right)
$$

with

$$
\begin{aligned}
& \theta_{1}=\phi+\pi\left(1+\text { floor }\left(\frac{\rho}{2 \pi a}\right)\right) \\
& \theta_{2}=\phi-\pi\left(1+\text { floor }\left(\frac{\rho}{2 \pi a}\right)\right)
\end{aligned}
$$

and $\phi=\operatorname{atan} 2(y, x), \rho=s \sqrt{x^{2}+y^{2}}, a=0.6, s=0.5$, into a sphere of radius 10 with uniform unit edge length. The surfacic adaptation and a volumic cut of the adapted meshes on 1 and 1024 processes are shown in figure 2. The resulting edge length statistics are shown in table 2. The strong scaling performances are shown in logarithmic scale in figure $1 \mathrm{~b}$, where the speedup $S_{p}$ over $p$ processes is

\footnotetext{
${ }^{1}$ Supported by Inria, CNRS (LABRI and IMB), Université de Bordeaux, Bordeaux INP and Conseil Régional d'Aquitaine (see https://www.plafrim.fr/)
} 


\begin{tabular}{|c||c|c|c|c||c|c|c|c|}
\hline$p$ & $n_{v}^{\text {in }} / p$ & $n_{v}^{\text {out }} / p$ & $n_{v}^{\text {out }} / n_{v}^{\text {in }}$ & $n_{v}^{\text {out }}$ & $n_{e}^{\text {in }} / p$ & $n_{e}^{\text {out }} / p$ & $n_{e}^{\text {out }} / n_{e}^{\text {in }}$ & $n_{e}^{\text {out }}$ \\
\hline 2 & 3625 & 1293637 & 356.81 & 2587274 & 18876 & 7780974 & 412.21 & 15561948 \\
4 & 3467 & 1341637 & 386.88 & 5366549 & 18798 & 8072081 & 429.39 & 32288325 \\
8 & 3346 & 1380055 & 412.38 & 11040444 & 18666 & 8306084 & 444.98 & 66448675 \\
16 & 3264 & 1412516 & 432.66 & 22600269 & 18599 & 8503695 & 457.2 & 136059129 \\
32 & 3190 & 1437267 & 450.46 & 45992552 & 18557 & 8654569 & 466.36 & 276946210 \\
64 & 3214 & 1431186 & 445.2 & 91595935 & 18625 & 8619098 & 462.75 & 551622317 \\
128 & 3215 & 1444674 & 449.31 & 184918370 & 18878 & 8701524 & 460.91 & 1113795077 \\
256 & 3345 & 1468905 & 439.01 & 376039759 & 19705 & 8848464 & 449.03 & 2265206835 \\
512 & 3375 & 1446532 & 428.52 & 740624790 & 19998 & 8714450 & 435.74 & 4461798709 \\
1024 & 3335 & 1449215 & 434.54 & 1483996788 & 19821 & 8731162 & 440.49 & 8940710661 \\
\hline
\end{tabular}

Table 1: Mesh statistics for the ParMmg weak scaling test. Number of vertices $n_{v}$ and tetrahedra $n_{e}$ in input and output using $p$ processors.

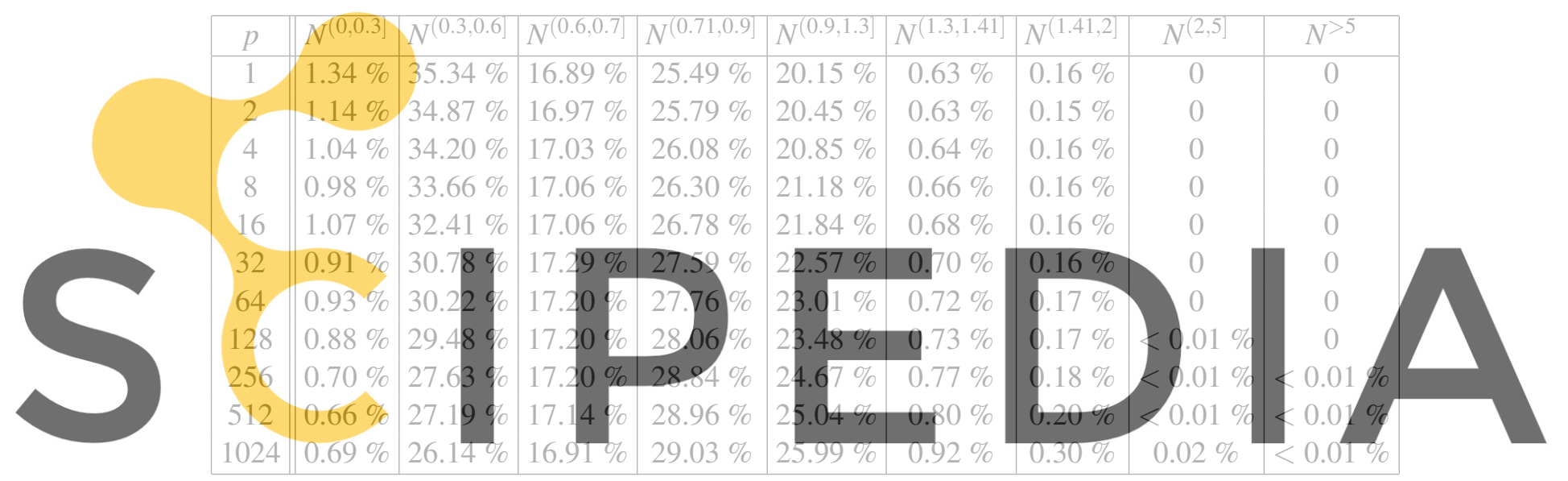

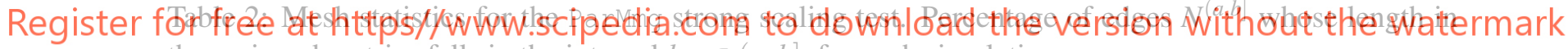
the assigned metrics falls in the interval $l_{\mathfrak{M}} \in(a, b]$, for each simulation on $p$ processors.

defined as the ratio between the wall time on 1 process and the wall time on $p$ processes

$$
S_{p}=\frac{T_{1}}{T_{p}}
$$

except for the speedup of the redistribution part of the program, which is defined with respect to the redistribution time on 2 processes instead of 1 (as there is no redistribution on 1 process). As the number of interfaces increases with the number of processes, it can be noticed both in table 2 and in figure $1 \mathrm{~b}$ that there is still a trend for a more pronounced propagation of large edge sizes from the fixed interfaces as the number of processes increases, as well as a performance reduction in the redistribution phase. These issues are the subject of current improvement efforts. 


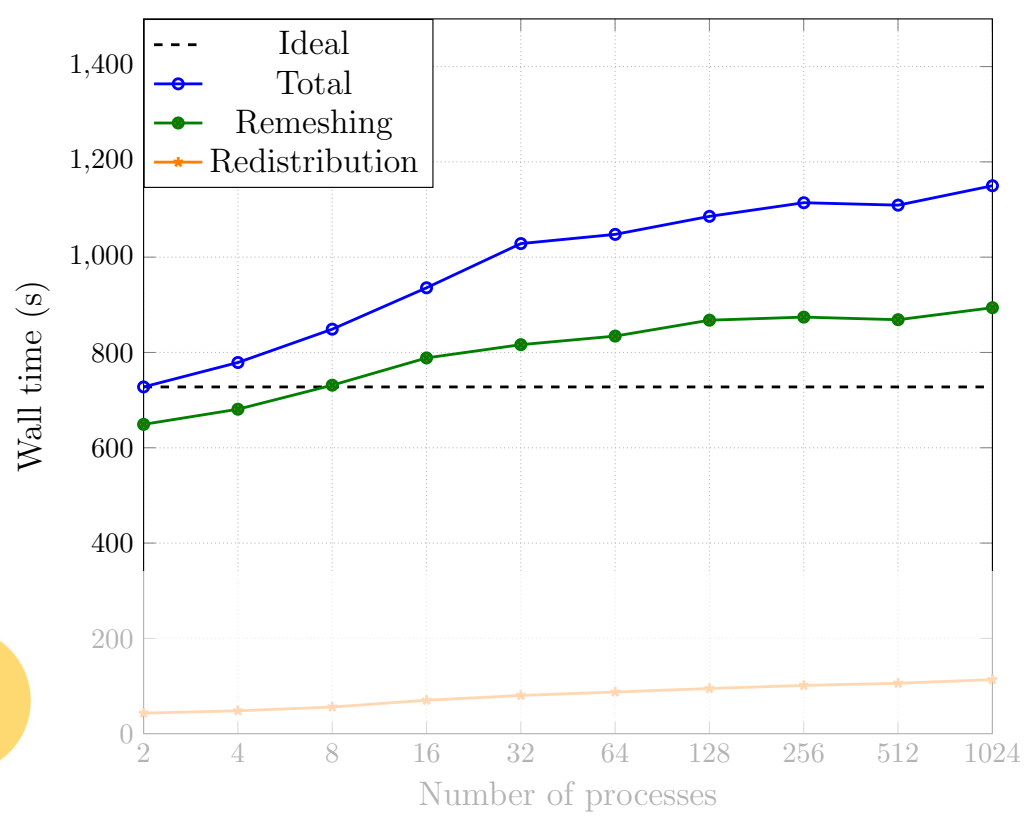

(a) Weak scaling performances for the uniform refinement of a sphere.
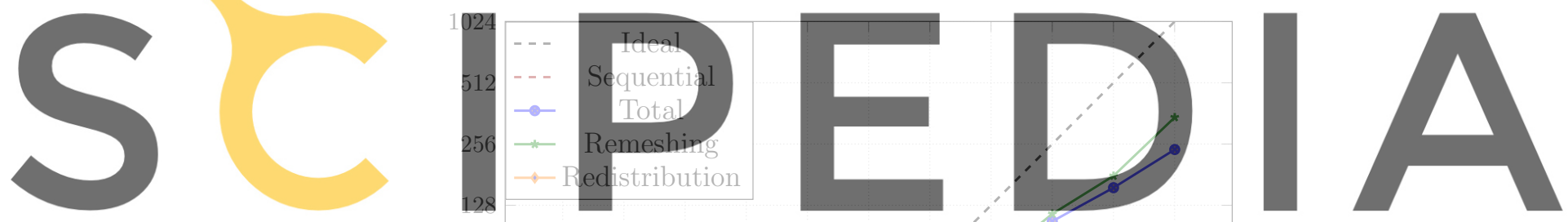

Register for free at httpsł/www.scipedia.com to downlóad the version without the watermark

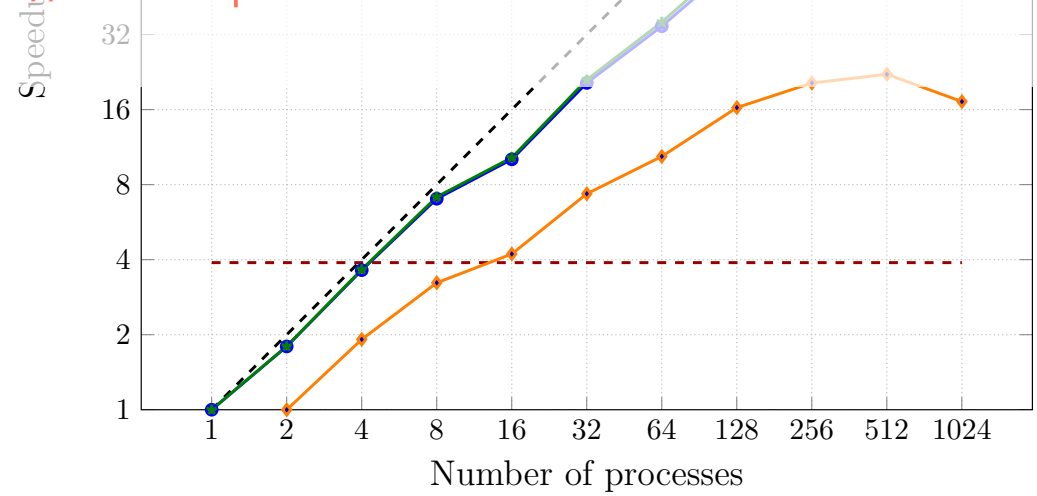

(b) Strong scaling performances for the double Archimedean spiral in a sphere.

Figure 1: Weak and strong scaling performances of ParMmg for two different tests. 

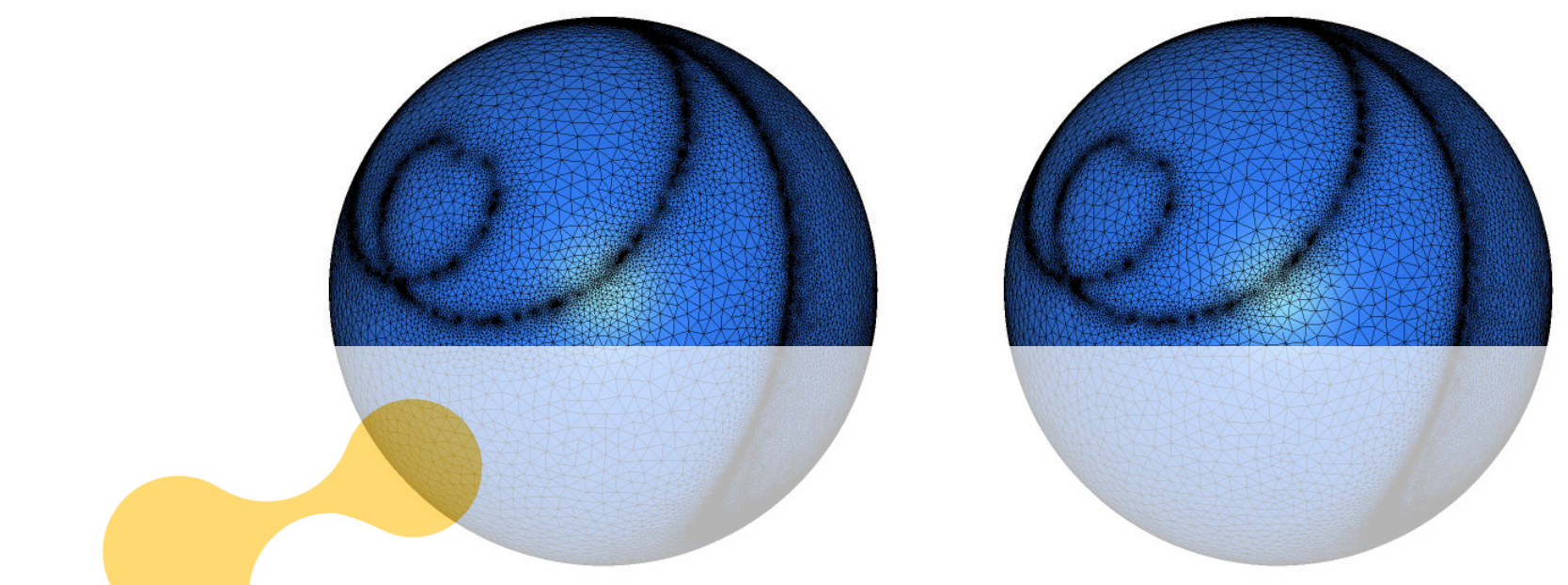

(a) Surface adaptation on 1 process.
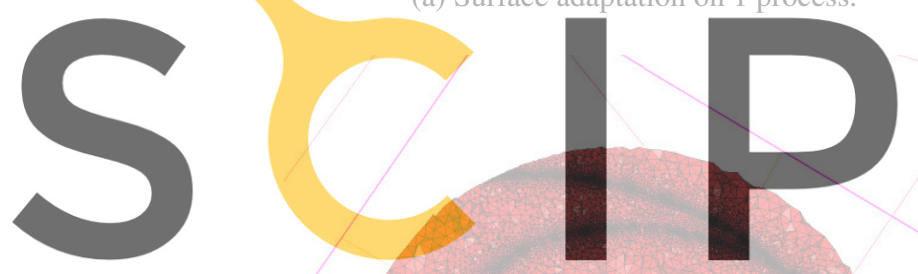

(b) Surface adaptation on 1024 processes.

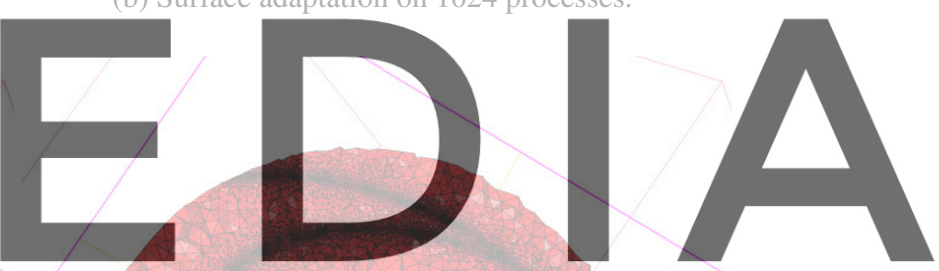

Register for free at https//www.scipedia.com to download the version without the watermark

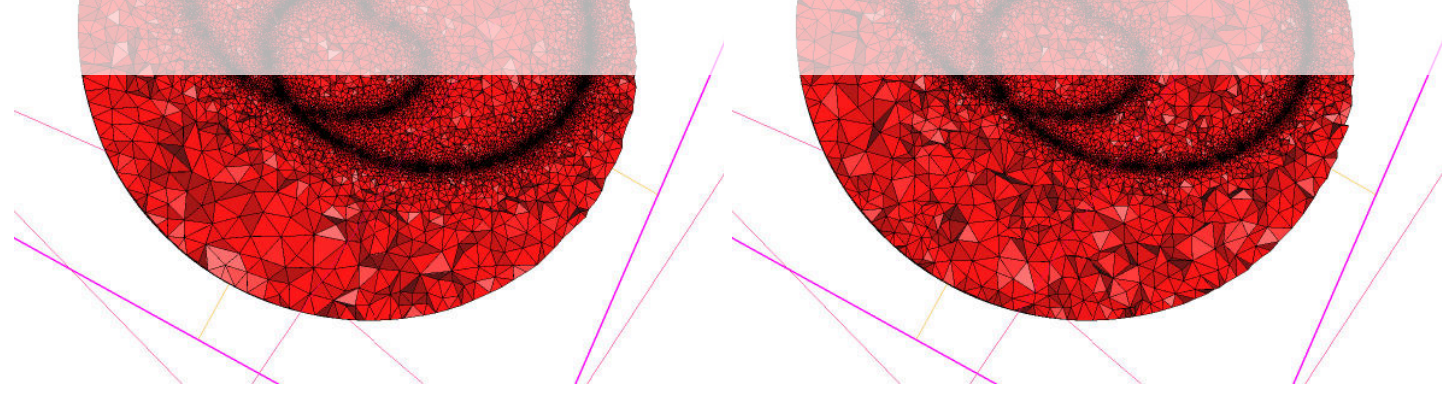

(c) Volume adaptation on 1 process.

(d) Volume adaptation on 1024 processes

Figure 2: Examples of adaptation to the double Archimedean spiral on 1 and 1024 processes. 


\subsection{Extension to anisotropic metrics}

As already shown in [12], the application to anisotropic metrics can suffer from a more serious size diffusion effect than the one shown in the previous section, if specific precautions are not taken when dealing with the metric specification on parallel interfaces. This is the subject of current studies.

\section{CONCLUSIONS}

We have shown a performance assessment of a parallel remeshing algorithm for unstructured meshes based on iterative remeshing-repartitioning first described in [12]. Parallel iterations are performed by alternating remeshing on interior process partitions, with fixed parallel interfaces, to mesh repartitioning aiming at moving previous interfaces on the interior of the next partitions to be remeshed. A key point for the choice of this specific parallelization strategy rests in software modularity, since building a parallelization framework on ton of an existing sequential remeshing kernel allows the parallel software application to benefit from the continuous development of its sequential kernel. Modularity also applies to the choice of the mesh repartitioning strategy, since it is independent from the internal mechanics of the remeshing kernel. While weak scaling results show that meshes of billions of tetrahedra are easily achievable through the current framework, they also show that care needs to be taken in the design of the parallel redistribution phase, as more data and more processes are involved in the communication phase. Strong scaling results show that the parallel application exhibits an overhead with respect to its sequential counterpart, due to the fact that several remeshing iterations are performed. Although this

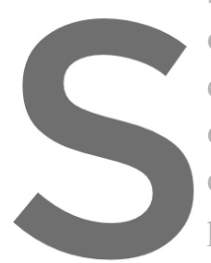
overhead is quickly catch coupling with an external on the performances of the cator size. Future research lines involve further imp to phase, which could be munication, as well as the reduction of the paralle
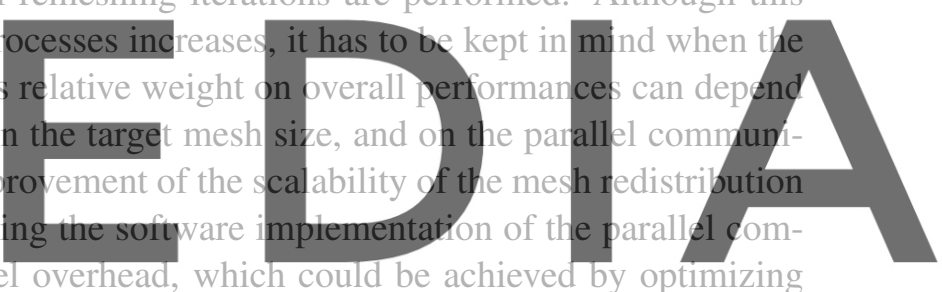
the number of iterations or the interface displacement phase. Future developments also concern the full Register for free athttpsthwWW scipedia com to dopwnlad the version without the watermark

\section{References}

[1] Michael A Park et al. "Unstructured Grid Adaptation: Status, Potential Impacts, and Recommended Investments Towards CFD 2030". In: AIAA Fluid Dynamics Conference, AIAA AVIATION Forum. Washington DC, United States, 2016. URL: https://hal. inria.fr/hal-01438667.

[2] José G. Castaños and John E. Savage. "The Dynamic Adaptation of Parallel Mesh-Based Computation". In: PPSC. 1997.

[3] H. L. De Cougny and M. S. Shephard. "Parallel refinement and coarsening of tetrahedral meshes". In: International Journal for Numerical Methods in Engineering 46.7 (1999), pp. 1101-1125.

[4] Leonid Oliker, Rupak Biswas, and Harold N Gabow. "Parallel tetrahedral mesh adaptation with dynamic load balancing”. In: Parallel Computing 26.12 (2000). Graph Partitioning and Parallel Computing, pp. 1583 -1608. URL: http://www. sciencedirect.com/science/article/pii/ S0167819100000478. 
[5] Nikos Chrisochoides and Démian Nave. "Parallel Delaunay mesh generation kernel". In: International Journal for Numerical Methods in Engineering 58.2 (2003), pp. 161-176. eprint: https: //onlinelibrary.wiley.com/doi/pdf/10.1002/nme.765. URL: https://onlinelibrary . wiley.com/doi/abs/10.1002/nme. 765 .

[6] J.E. Flaherty et al. "Parallel structures and dynamic load balancing for adaptive finite element computation”. In: Applied Numerical Mathematics 26.1 (1998), pp. 241 -263. URL: http: // www.sciencedirect.com/science/article/pii/s0168927497000949.

[7] Peter A. Cavallo, Neeraj Sinha, and Gregory M. Feldman. "Parallel Unstructured Mesh Adaptation Method for Moving Body Applications". In: AIAA Journal 43.9 (2005), pp. 1937-1945. eprint: https://doi.org/10.2514/1.7818. URL: https://doi.org/10.2514/1.7818.

[8] Hugues Digonnet et al. "Massively parallel anisotropic mesh adaptation". In: International Journal of High Performance Computing Applications (Mar. 2017). URL: https: / / hal . archivesouvertes.fr/hal-01487424.

[9] Pierre Benard et al. "Mesh adaptation for large-eddy simulations in complex geometries". In: International Journal for Numerical Methods in Fluids 81.12 (2016), pp. 719-740. eprint: https:// onlinelibrary.wiley.com/doi/pdf/10.1002/fld.4204. URL: https://onlinelibrary . wiley.com/doi/abs/10.1002/fld.4204.

[10] ParMmgversion 1.3.0. SWHID:swh:1:rel:bf45d6314a386455d53a6c351be771d6252e0d43; REPOSITORY: https://github.com/MmgTools/ParMmg.

[11] Mmg version 5.5.2. swHID: swh:1:rel:fe173a75f45f079d363d5a82204c9737550c5d79; REPOSITORY: https://github.com/MmgTools/mmg.

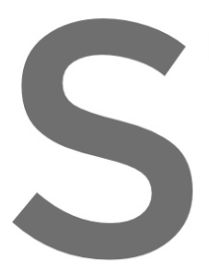

[12]
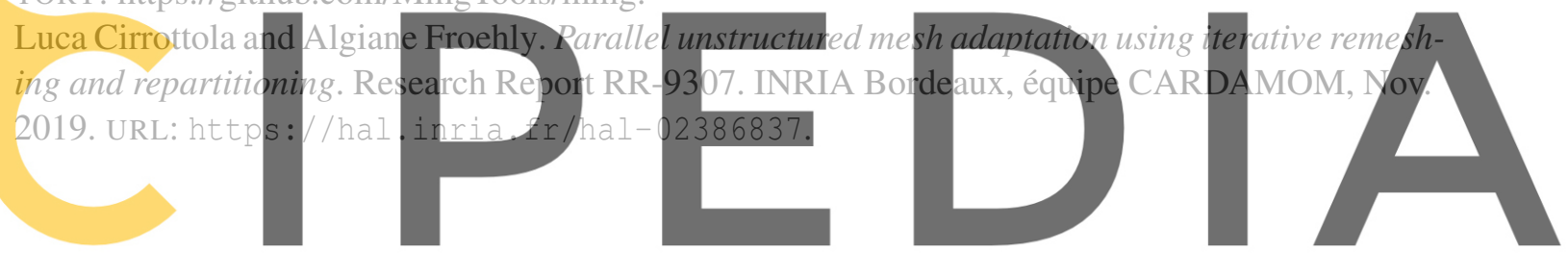

Register for free at https//www.scipedia.com to download the version without the watermark 